Whiте, H. R. (1953). J. gen. Microbiol. 8, 27-37.

\title{
The Heat Resistance of Streptococcus faecalis
}

\author{
By HELEN R. WHITE \\ Department of Hygiene and Bacteriology, King's College of \\ Household and Social Science, London, W. 8
}

SUMMARY: The log survivor/time graph for mature cultures of one strain of Streptococcus faecalis was not always straight over its whole length. A preliminary period of slow death was frequently observed, and a final similar period less often. The log survivor/time graphs of young cultures varied in shape with age of culture and often showed an initial rapid death-rate, passing suddenly into a slower one.

The heat resistance of three strains was considerably influenced by the age of the culture. Resistance, judged either by the Decimal Reduction Time or the time taken to reach $99.9 \%$ mortality, was increased by transfer to fresh medium and fell during the lag phase of growth, reaching a minimum as rapid reproduction began. A second maximum in heat resistance occurred when growth became slow: thereafter the resistance gradually fell to that of mature cultures. The incubation temperature affected the heat resistance of young cultures, the effect of high temperatures being particularly marked and resulting in increased resistance, except with cultures $1 \mathrm{hr}$. old or less.

The heat resistance of the faecal streptococci has been the subject of much investigation and discussion ever since Houston \& McCloy (1916) laid such stress on the ability of their strain to withstand heat. Nearly all measurements of resistance have been by 'end-point' methods, which are open to criticism because they place undue emphasis on a few highly resistant cells which are not typical of the population as a whole. A study of the death-rate of the majority of the cells in a culture should provide a better indication of thermal resistance.

Richards \& White (1949) showed that the resistance of Streptococcus faecalis to moist heat at $60^{\circ}$ may be conveniently expressed by the Decimal Reduction Time (DRT) of Katzin, Sandholzer \& Strong (1943) which is obtained from the relationship between the logarithms of the survivors and time. Fairly consistent DRT values of approximately $1 \mathrm{~min}$. were obtained with various strains, but the death-rate was not always constant. The present paper presents the results of further investigations on these lines.

\section{METHODS AND MATERIALS}

General technique. The technique used throughout this work was that described by Richards \& White (1949). Briefly, a standardized number of cells was added to a flask of saline held in a water-bath at $60^{\circ}$ and the survivors determined in samples taken from this flask at timed intervals. The fixed number of cells was obtained by centrifuging glucose Yeastrel broth cultures and resuspending the deposit in saline to a standard turbidity. Counts of survivors were obtained by the plating method using glucose Yeastrel agar, two or three plates being made of each dilution. 
Cultures. The strains of Strep. faecalis used were isolated, either from sour milk, human faeces or canine faeces, by the author. Six authentic strains kindly supplied by Dr P. M. F. Shattock were also used. Three strains were studied most, one (L5) from human faeces, one (L6) from canine faeces; the third strain ( $\mathrm{C}$ and $\mathrm{G}$ ), from milk powder, was supplied by Dr Shattock. The cultures were characterized by morphological and biochemical tests, and their identity confirmed by these tests at intervals. They were maintained in cooked meat medium, in which they were transferred every 3 months. Cultures for heat resistance tests were grown in glucose Yeastrel broth $(1 \%$ peptone, $\mathbf{0 . 2 5} \%$ sodium chloride, $0 \cdot 3 \%$ Yeastrel, $1 \%$ glucose, $\mathrm{pH} \mathbf{7 \cdot 2}$ ) under the required conditions.

Young cultures. Very young cultures needed to be inoculated into the broth more heavily than usual to give, during the short incubation, sufficient cells for an experiment. Various trials were made to find the lowest possible inoculum, it being clearly desirable to keep this small to ensure that the number of older cells in the standard suspension should be as low as possible. Eventually $0.1 \mathrm{ml}$. portions of parent $(18 \mathrm{hr}$.) cultures were inoculated into glucose Yeastrel broth: when necessary the deposits from several tubes were combined.

Incubation. To ensure accuracy in timing short periods of incubation, a water-bath controlled to $\pm 0 \cdot 5^{\circ}$ was used and the broth tubes brought to the proper temperature in the bath before inoculation.

When the effect of alteration of incubation temperature was being studied, the question of the temperature at which to incubate the parent culture arose. Ellicker \& Frazier (1938) found that the incubation temperature of the parent cultures greatly influenced the resistance of Bacterium coli, especially during the lag phase of growth, in which $28^{\circ}$ cultures inoculated from a $38.5^{\circ}$ parent culture showed greater resistance than did $38.5^{\circ}$ cultures inoculated from parents at either 38.5 or $28^{\circ}$. It was finally decided to incubate the parent culture at its optimum $\left(37^{\circ}\right)$ at this stage of the work so that effects due to varying incubation temperature would have been produced during one subculture only.

Direct microscopic counts. Two separate wire loopfuls were removed vertically from the incubating broth culture and spread over separate 1 sq.cm. areas on different slides. The smears were then dried quickly, fixed and stained. The average number of units in a microscope field was then obtained by counting five to ten fields on each slide, groups and individual cells being each treated as units. It was noted that the size of the groups varied greatly with the age of the culture, but the results from the two slides generally agreed well.

Jennison (1937) with Bact. coli concluded that, as the amount of clumping varied, the plate count could not bear a constant relationship to the total count. Obviously, the relationship must be far more variable with chainforming organisms such as streptococci, but counting both groups and individual cells as units should reduce the discrepancy between the results of the two methods, and the direct count had the advantages of speed and economy in materials. 
Estimation of resistance. The Decimal Reduction Time (DRT) was obtained from graphs on which the logarithms of the survivors were plotted against time. The steepest straight part of the line was always used for calculation purposes, so any preliminary periods of slow death or any tailing-off periods towards the end were not allowed for in the DRT values. It was realized that values so obtained would not take into account the characteristic features of some curves but their use was felt to be justified for the purposes of comparison.

To give a more complete picture of resistance in cases where the logarithmic death-rate varied during an experiment, 99.9\% mortality times were also obtained. These were read off from the log survivor/time graphs, using the count of the first sample as the reference value. Since the experiments were originally designed to yield information about variations in DRT only, it had not been envisaged that counts of the numbers of organisms added to the saline in the flask would be required.

Assessment of initial and final periods of slower death. The steepest straight portion of the log survivors/time graph was extrapolated backwards, and the time at which this line reached the level of the count in the first sample (usually about $\log =7$ ) was taken as the duration of the initial period of slow death. The final period of slow death was assumed to begin where the experimental data indicated a break from the portion of steepest slope.

\section{RESULTS}

\section{The shape of the death-rate curve}

It has already been shown that the log survivor/time relationship for Strep. faecalis exposed to heat at $60^{\circ}$ is not always linear (Richards \& White, 1949). In many cases an initial portion of small slope is observed, the slope increasing gradually to a maximum which is usually maintained until the end of the test, though there may be a resistant tail of organisms whose death-rate is slower. Preliminary periods of slow death are important and must be borne in mind when comparing the heat resistance of various organisms. Some means of indicating its occurrence and duration should be included in any expression of heat resistance, and its frequent appearance with Strep. faecalis strains seemed to merit more detailed study.

Mature cultures. A preliminary survey was made of twenty experiments which were selected because they were all done within a few days on mature cultures of strain L 5 incubated at $37^{\circ}$. Records of the incidence and duration of initial periods of slow death and the time of onset of tail periods showed that in three cases there was no initial period of slow death at all, fourteen had slow periods up to $2 \mathrm{~min}$. and three slow periods up to $3 \mathrm{~min}$. There was no tail in fourteen cases, a tail from $4 \mathrm{~min}$. in five cases and a tail from $6 \mathrm{~min}$. in one case. From this it was concluded that while an initial period of slow death of just over 2 min. was usually present, a tail period occurred less often, and when present did not appear until at least $4 \mathrm{~min}$. after the beginning of the experiment. 
Length of period of slow death in mature cultures. As all the above experiments were carried out with samples taken at the end of the first minute after subjection to heating and at $1 \mathrm{~min}$. intervals thereafter, it was realized that more exact information on the duration of the period might be obtained by staggering the sampling times in a series of experiments. The sampling was still at $1 \mathrm{~min}$. intervals but the time of taking the first sample was varied. Twelve successive experiments of this type on strain L 5 gave a mean duration for the initial period of slow death as $\mathbf{2 \cdot 2 5}$ min.

A survey of the incidence and length of this period of slow death with other strains of Strep. faecalis exposed to heat at $60^{\circ}$ was also made, and it was concluded that, although not always present, it occurs frequently. It was almost invariably shown by strains L5 and 61, and also by the six strains supplied by Dr Shattock. Strains L 6 and 62 showed it sometimes and strain 63 hardly ever. Strain 64 was abnormal, the death-rate being very slow : although followed for 25-30 min., no change in rate could be detected.

Young cultures less than $12 \mathrm{hr}$. old. These gave very variable results and the exact age of the culture was important. Fig. 1 shows log survivor/time graphs for strain L 5 at $37^{\circ}$. The culture $\mathbf{3 0}$ min. old was highly but uniformly resistant while the $\mathbf{2 . 5} \mathrm{hr}$. culture showed an initial fast death-rate which changed suddenly to a lower value. Fig. 2 illustrates the behaviour of strain L6. Cultures $1 \mathrm{hr}$. old showed an initial period of slower death followed by one of constant faster death, while the $\mathbf{1 . 5} \mathrm{hr}$. culture showed an initial rush and sharp break. With the other strains the break was often less sudden and the graph approximated more to a curve of gradually lessening slope. Thinking that the variability might have been due to heterogeneity in the young cultures, i.e. that cells which had not yet succeeded in dividing were mixed with those in active division, an attempt was made to make the population more uniform by subculturing serially after incubation for $2 \mathrm{hr}$. Fig. 3 shows the result using strain $\mathrm{L} 5$, with organisms from the fourth subculture at $37^{\circ}$. The death-rate is uniform.

\section{The influence of age of culture on DRT}

Strain L5; 12-48 hr. cultures. Fifty-two experiments with $22 \mathrm{hr}$. cultures of strain L 5 gave a mean DRT of $1.02 \mathrm{~min}$. The values from cultures 12, 18, 36 or $48 \mathrm{hr}$. old (shown in Table 1) were not significantly different from this. This finding may be compared with that of Stark \& Stark (1929) who observed that the resistance of Strep. faecalis cultures increased up to $24 \mathrm{hr}$. after which it decreased up to $48 \mathrm{hr}$.

Strain L5; cultures less than $12 \mathrm{hr}$. old. Replicate experiments (usually five or six, never less than three) were carried out on cultures $0 \cdot 5-8 \mathrm{hr}$. old. The DRT's varied somewhat from experiment to experiment, but Fig. 4 shows how the mean value of the DRT varied with the age of the culture within this period. It may be seen that the youngest cultures $(30 \mathrm{~min}$. old) were much more resistant than the parents, having a mean DRT of $3 \cdot 1 \mathrm{~min}$. against about $1 \mathrm{~min}$. for the parent cultures. There was a further slight rise in resistance for the next $30 \mathrm{~min}$. of incubation, after which a steep decline set in, a minimum 
value being reached at about $2 \mathrm{hr}$. This was followed by a gradual rise to a second but less high peak after about $5.5 \mathrm{hr}$. Later there was a gradual fall in resistance to the figure of about $1 \mathrm{~min}$. at $8 \mathrm{hr}$, , and the figure was still approximately the same at 12, 18, 36 and $48 \mathrm{hr}$. as already shown.

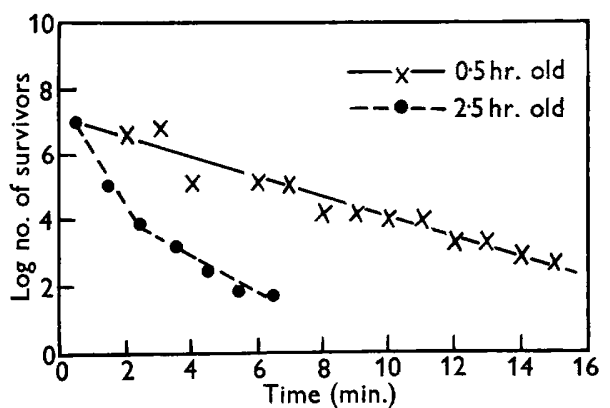

Fig. 1

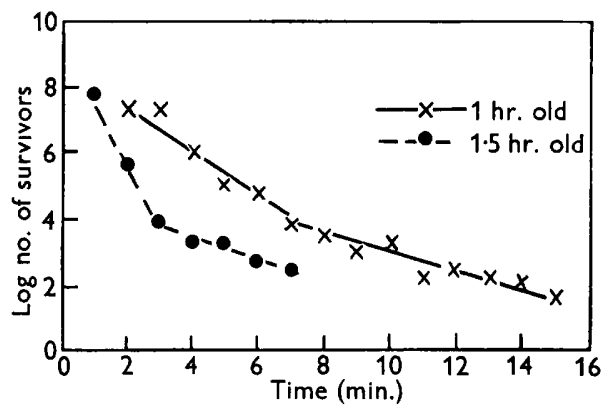

Fig. 2

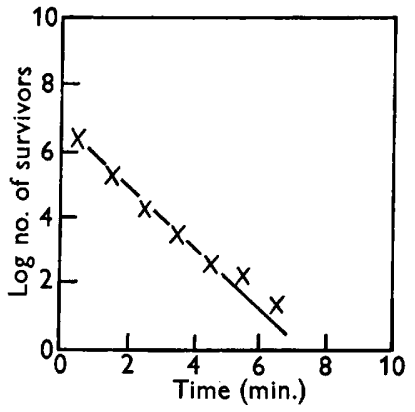

Fig. 3

Fig. 1. The log survivor/time graphs for typical young cultures of Strep. faecalis strain L 5 exposed to heat in saline at $60^{\circ}$ (incubation at $37^{\circ}$ ).

Fig. 2. The log survivor/time graphs for typical young cultures of Strep. faecalis strain L 6 exposed to heat in saline at $60^{\circ}$ (incubation at $37^{\circ}$ ).

Fig. 3. The log survivor/time graph of a 'built-up' young culture (four successive $2 \mathrm{hr}$. subcultures) of Strep. faecalis (strain 'L5') at $60^{\circ}$ in saline.

Table 1. Effect of age of culture on resistance of Strep. faecalis (strain L5) incubated at $37^{\circ}$

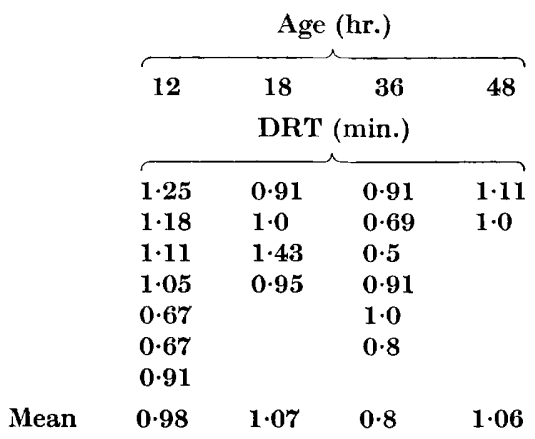


Results with other strains. Young cultures of strain $\mathrm{L} 6$ and strain $\mathrm{C}$ and $\mathrm{G}$ gave resistance curves of the same general shape as that for $\mathrm{L} 5$, though they varied in detail. With strain $\mathbf{L} 6$, the first stage of greater resistance lasted longer and the point of least resistance was delayed until about $3 \mathrm{hr}$. The second peak of resistance occurred between 6 and 9 hr., i.e. the whole cycle of resistance took longer to accomplish. With strain $\mathbf{C}$ and $\mathbf{G}$ the first stage of increased resistance was shorter than with $\mathrm{L} 5$, and after only $1 \mathrm{hr}$. of incubation the point of minimum resistance was reached. The second peak was also reached slightly sooner, in about $4.5 \mathrm{hr}$.

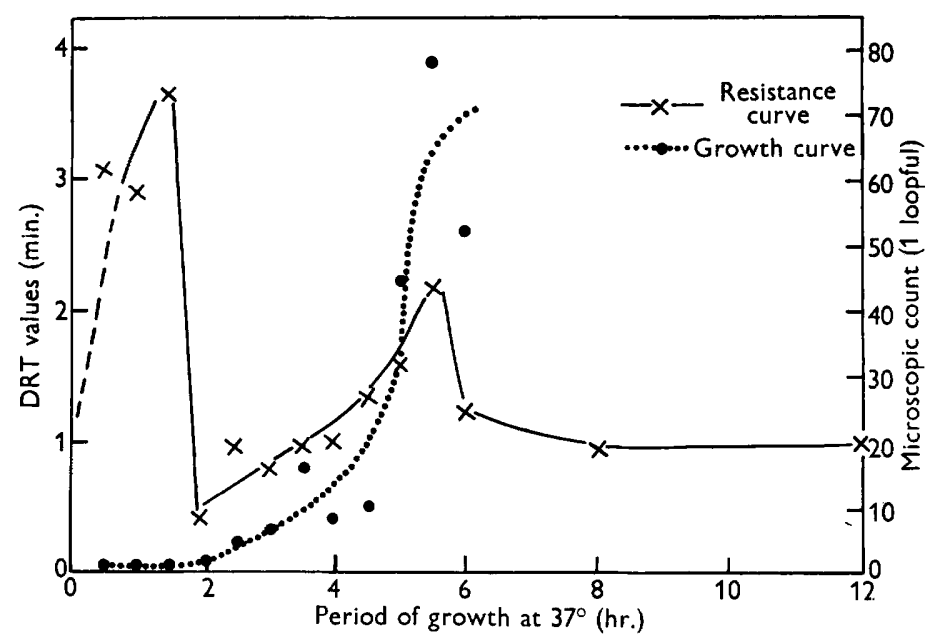

Fig. 4. The effect of the age of the culture on the resistance (expressed as DRT) of Strep. faecalis strain L5 at $60^{\circ}$ in saline (incubation at $37^{\circ}$ ).

The relation between resistance and growth phase. To see whether this variation in timing but not in general shape of the resistance curves was associated with differences in the growth-rates of the different strains, the development of broth cultures was followed by means of direct microscopic counts. Several experiments were carried out and the average counts plotted against time of incubation. The growth curve for strain L5 is shown in Fig. 4 together with the resistance curve.

There was a marked association between the phases of the growth curves (inaccurate as they were) and those of the resistance curves, especially with strain L 5. It will be seen that during the lag period of growth this organism showed its greatest resistance to heat. As soon as active reproduction commenced, or possibly slightly before, resistance fell to the minimum. As growth proceeded through the logarithmic phase resistance rose again and may have reached the second peak at the end of that phase, but counts were not carried on sufficiently long for this to be established definitely.

One of the more interesting features of these age/resistance curves is the spectacular rise in resistance of the very young (lag phase) cultures. Within a short time of subculturing to fresh medium (as little as $15 \mathrm{~min}$. with some 
strains) the resistance had at least doubled. Further investigations into this point are planned.

With all three strains it was noted that cultures up to $4 \mathrm{hr}$. old had more large groups and chains than the older cultures. Probably a temporary increase in chain length is an indication of rapid multiplication. For this reason, the microscopic counts cannot be said truly to portray the increase in cell numbers with time; but the same criticism would apply to plate counts had they been done instead. However, the observation of this increase in chain length is of some importance as it may explain to some extent the less regular death-rates of these young cultures.

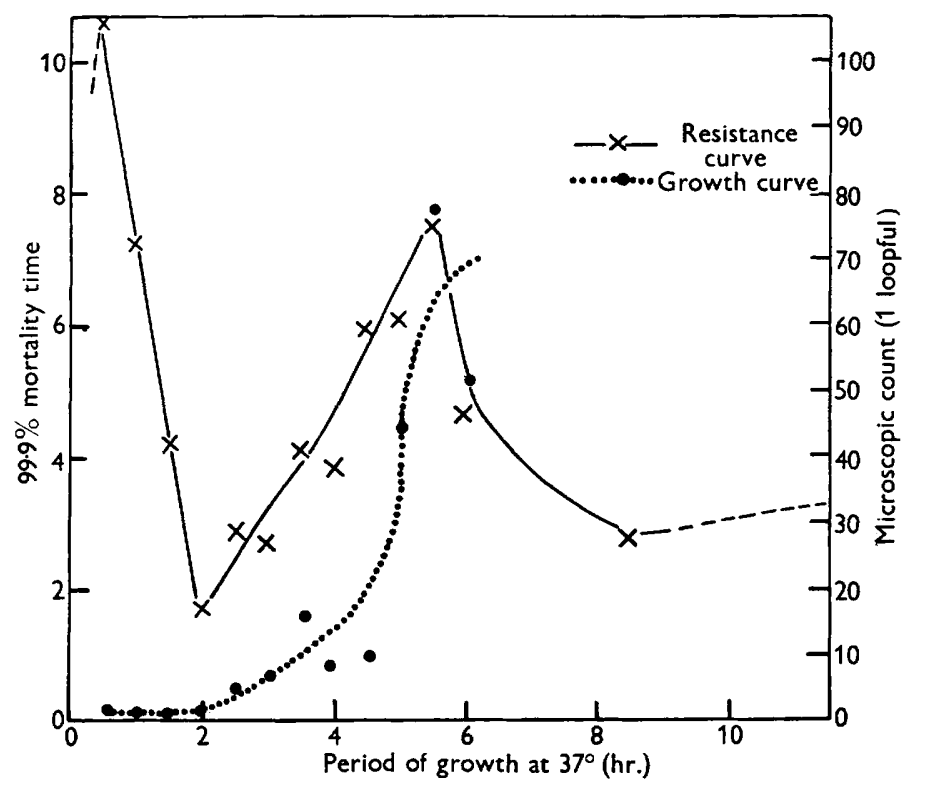

Fig. 5. The effect of the age of the culture on the resistance (expressed as $99.9 \%$ mortality time) of Strep. faecalis strain L 5 at $60^{\circ}$ in saline (incubation at $37^{\circ}$ ).

\section{The influence of age on mortality time}

It is evident that when resistance is estimated in terms of the DRT which is calculated from the steepest portion of the log survivor/time curve, the estimates err by not taking into consideration the length of any preliminary period of slow death. This is especially important with very young cultures, where the logarithmic death-rate is by no means constant. Accordingly, $\mathbf{9 9 . 9} \%$ mortality times were obtained from the same curves. Naturally, variations in the shapes of different curves influenced the results considerably, but the general relationship between age and resistance was not altered. This may be seen from Fig. 5 where the results for strain L 5 are shown. Similar results were also given by strains $\mathrm{L} 6$ and $\mathrm{C} 9$, and it may be concluded that the resistance of young cultures of Strep. faecalis does indeed vary in a constant manner according to the growth phase. 


\section{The influence of incubation temperature on the resistance of young cultures}

Strain $\mathbf{L}$. The results of experiments carried out with young cultures of strain $\mathbf{L} 5$ incubated at $27^{\circ}$ are shown in Fig. 6. The general pattern of changes in resistance was the same as at $37^{\circ}$, and there was clearly the same association with the growth phases. The lag phase was longer (about $4 \mathrm{hr}$. instead of $2 \mathrm{hr}$. as at $37^{\circ}$ ) and resistance fell steadily as it progressed, reaching a minimum

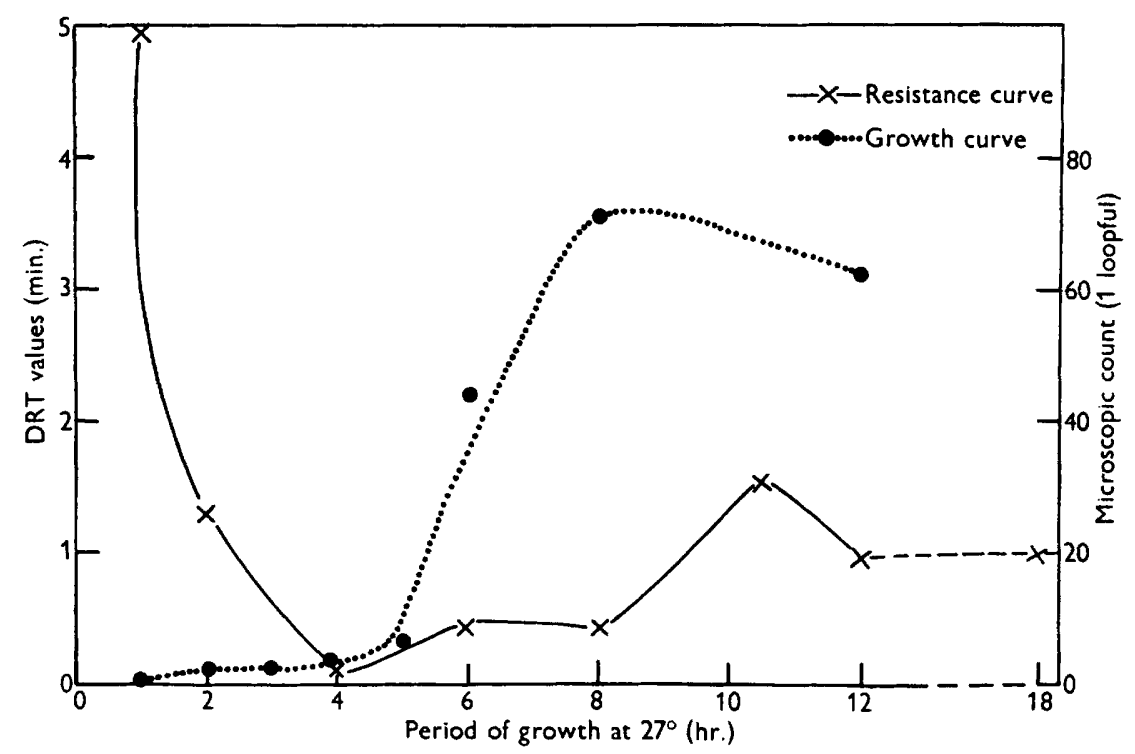

Fig. 6. The growth curve and resistance curve of young cultures of Strep. faecalis strain $\mathrm{L} 5$ incubated at $27^{\circ}$.

just as active growth began. The whole cycle of changes took far longer at $27^{\circ}$ than at $37^{\circ}$, due to the slower growth rate. However, the difference between the maximum and minimum resistance was much greater. During the lag phase there was a higher DRT than at $37^{\circ}$, but when the resistance dropped, as the logarithmic phase began, it fell much below the value for $37^{\circ}$. It is possible that the apparently exaggerated changes in the resistance at $27^{\circ}$ came about, in part, because it was easier to obtain truly representative cultures of each age when the rate of growth was slower. After the minimum value the DRT rose slowly to a minor peak in $10 \mathrm{hr}$. cultures and subsequently declined to about 1 min., at which level it remained constant. The final condition was thus the same as in mature cultures at $37^{\circ}$.

At $45^{\circ}$ also, the general shape of the resistance curve was the same as at $37^{\circ}$ (Fig. 7), but the point of minimum resistance was reached at about $5 \mathrm{hr}$. The direct microscopic counts of the $45^{\circ}$ cultures were not very satisfactory. since the organisms tended to form into large clumps and tangled chains which were sometimes clearly visible to the naked eye as large easily dispersed 
flakes. However, it seemed that resistance rose to a peak in the lag phase and fell sharply when this ended, only to rise again as the logarithmic phase progressed.

The outstanding feature of the $45^{\circ}$ results is that the DR'T values were above those for cultures of the same age incubated at 27 and $37^{\circ}$, except for the very youngest cultures. The final resistance in the stationary phase was

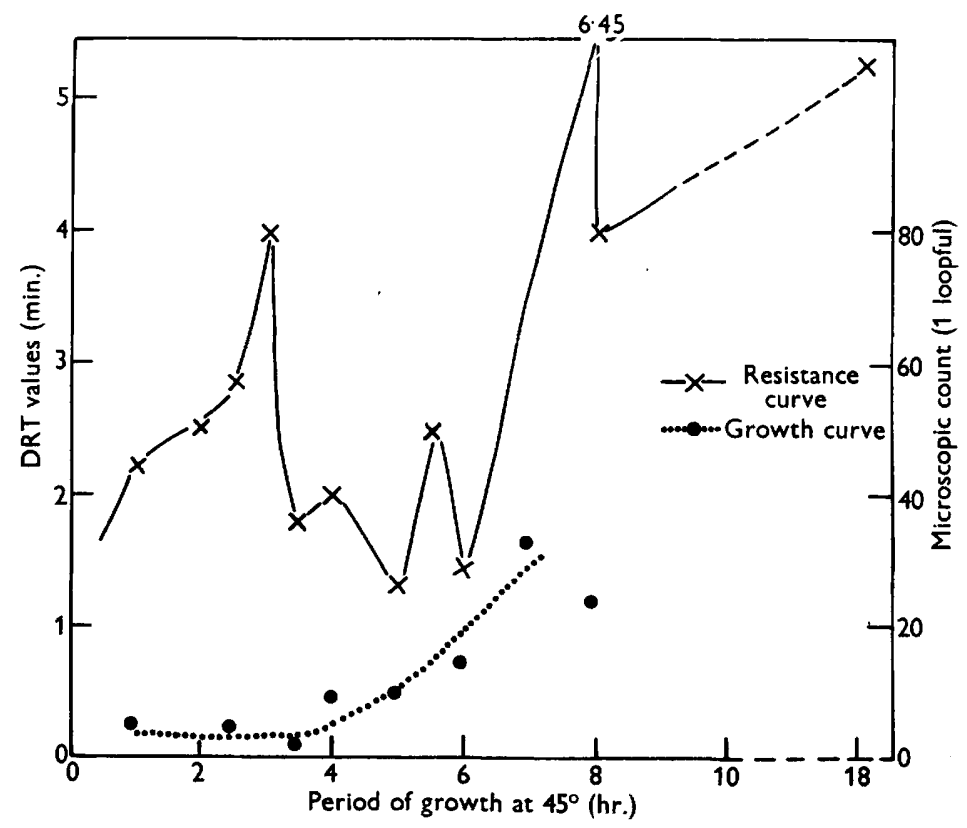

Fig. 7. The growth curve and resistance curve of young cultures of Strep. faecalis strain $\mathrm{L} 5$ incubated at $45^{\circ}$.

very great indeed, and was retained at this high level until at least $18 \mathrm{hr}$. Thus, the mature cells in $45^{\circ}$ cultures were more resistant than mature cells from 27 and $37^{\circ}$ cultures. This unexpected finding agrees with the results of Ellicker \& Frazier (1938). Possibly the tendency to form larger groups at the higher incubation temperature may partly explain the higher resistance.

Other strains. Strains L 6 and strain $\mathrm{C}$ and $\mathrm{G}$ behaved like L5 in their general pattern of resistance, showing minor differences, as was to be expected. It was immaterial whether the resistance was expressed as the $99.9 \%$ mortality time or in terms of the DRT. At $45^{\circ}$ both $\mathrm{L} 6$ and $\mathrm{C}$ and $\mathrm{G}$ showed the much enhanced resistance that was so marked a feature of the $\mathbf{L} \mathbf{5}$ cultures at this temperature.

\section{DISCUSSION}

There is a striking similarity between the results reported above for Strep. faecalis, and those of Ellicker \& Frazier (1938) with Bact. coli, in spite of the great difference between these organisms in type and habit of growth. Strep. 
faecalis forms aggregates more frequently than Bact. coli; this property was expected to confuse the present results to some extent. It may have done so, particularly in the initial period of slow death which may have been an artefact due to the presence of clumps, though Jordan \& Jacobs (1944) have clearly established that such a period is shown even by Bact. coli when the death-rate is made sufficiently slow for samples to be secured before the acceleration has set in. The existence of variations in death-rate during an experiment may be due, as suggested by Hinshelwood (1951), to variations in the resistance of cells according to the stage they have reached in the division cycle.

The present results differ from those of Ellicker \& Frazier (1938) only in that lowered temperature did not lead to lowered resistance at all stages of growth. Very young cultures of Strep. faecalis at $27^{\circ}$ were more resistant than those at $37^{\circ}$, and those at $37^{\circ}$ were slightly more resistant than those at $45^{\circ}$. The marked increase in resistance which occurred very soon after transference to fresh medium was striking, as was the sharp fall at the onset of logarithmic growth. In view of these great variations within a short period, and the subsequent changes, it is suggested that the apparently conflicting results of earlier workers, many of whom found decreased resistance in young cultures while a few observed enhanced resistance, may be attributed to differences in technique. Evidently the term 'young culture' needs rigid definition. It is also likely that in different media the time relationships may alter even with one organism, while different organisms would undoubtedly take different times to reach the several phases. Although Ellicker \& Frazier's results have been matched so closely on the present work, those workers' curves, both for resistance and growth, show that Bact. coli grown in milk took longer to accomplish the full cycle than did Strep. faecalis grown in broth.

I am particularly grateful to Dr S. E. Jacobs of Imperial College, London, for his immense help in the preparation of this paper. I would also like to thank Dr T. Richards of the University of Reading for his interest and advice throughout the work and Mrs Atkins, the Librarian of King's College of Household and Social Science, for her help in obtaining literature.

\section{REFERENCES}

Ellicker, P. R. \& Frazier, W. C. (1938). Influence of time and temperature of incubation on heat resistance of Escherichia coli. J. Bact. 36, 83.

Hinshelwood, C. (1951). Decline and death of bacterial populations. Nature, Lond. 167, 666.

Houston, T. \& McCloy, J. M. (1916). The relation of the enterococcus to trench fever and allied conditions. Lancet, ii, 632.

JENNison, M. W. (1937). Relations between plate counts and direct microscopir counts of Escherichia coli during the logarithmic phase. J. Bact. 33, 461.

Jordan, R. C. \& JacoBs, S. E. (1944). Studies in the dynamics of disinfection. II. The calculation of the concentration exponent for phenol at $35^{\circ}$ with Bact. coli as test organism. J. Hyg., Camb. 43, 6, 363. 
Katzin, L. I., Sandholzer, L. A. \& Strong, M. E. (1943). Application of the decimal reduction time principle to a study of the resistance of coliform bacteria to pasteurization. J. Bact. 45, 265.

Richands, T. \& White, H. R. B. (1949). The heat disinfection of Streptococcus faecalis. Proc. Soc. app. Bact. 1949, no. 2, 61.

Stark, C. N. \& Stark, P. (1929). The relative thermal death rates of young and mature bacterial cells. J. Bact. 18, 333.

(Received 4 March 1952) 